\title{
In Situ TEM Study of Friction and Wear of Olivine
}

\author{
Sanjit Bhowmick ${ }^{1 *}$, Eric Hintsala ${ }^{1}$, Douglas Stauffer ${ }^{1}$ and SA Syed Asif ${ }^{1}$ \\ ${ }^{1 .}$ Bruker Nano Surfaces, Minneapolis, MN, USA. \\ * Corresponding author: sanjit.bhowmick@bruker.com
}

The study of tribology includes wear, friction, and lubrication of interacting surfaces in relative motions. Since the function, efficiency, and lifetime of many engineering components depends on the appropriate friction and wear properties, the study of tribology has significant practical importance. The fundamental knowledge to control friction and wear behavior is applicable to mechanical and electromechanical systems as well as to biological structures. However, traditional tribology tests are limited by the inability to observe real-time progress of the sliding contacts and wear mechanism. Recent developments of small-scale devices, particularly piezoelectric and MEMS-based actuators, aid in conducting scratches at the micro- and nano-scale in the TEM [1-3]. The real-time observation may help in understanding surface energy, elastic and elasto-plastic deformation, formation of micro-cracks, and surface interactions.

In this study, a 2D MEMS transducer was used to conduct in situ tribology tests of an olivine sample. MEMS actuators can provide high force- and displacement-resolution and good thermal stability which are important factors to obtain precise mechanical properties and high-resolution images. The transducer used in this study (Bruker Nano Surfaces, Minneapolis, MN, USA) consists of two electrostatic micromachined comb drives in a single body that actuate and sense force and displacements in normal and lateral directions simultaneously. A diamond tip was coupled to a comb drive that actuates a motion towards indentation axis and the second comb drive comprising a plurality of electrostatic capacitive actuators drives the same probe perpendicular to the indentation axis. This 2D MEMS transducer was used with a TEM nanomechanical instrument, Hysitron PI 95 (Bruker Nano Surfaces, Minneapolis, MN, USA), inside Tecnai G2 F30 TEM (Thermo Fisher Scientific, Hillsboro, OR, USA).

Olivine is magnesium iron silicate $\left(\mathrm{Mg}^{2+}, \mathrm{Fe}^{2+}\right)_{2} \mathrm{SiO}_{4}$ and the most abundant mineral in earth's upper mantle, which comprises the bulk of the planet's tectonic plates. Although the structure of olivine has been intensively studied by mineralogists and geophysicists, the frictional and mechanical properties particularly damage evolution at the small-scale of sliding contact is unknown. The tribological properties of olivine are important to understand plate tectonics and the movement of plates in subduction zones of earth. In this study, a bulk olivine sample was wedge polished and then fib-milled to prepare electron-transparent regions. A wedge diamond indenter of $100 \mathrm{~nm}$ radius and 3 um length was used to make sliding contact. A normal load was kept on the sample surface while the tip was moved a predetermined lateral distance with a particular rate. Multicycle scratch tests were conducted to understand wear behavior. Fig. 1a plots the data from a multicycle scratch test where normal loads of 50 $\mu \mathrm{N}, 98 \mu \mathrm{N}, 142 \mu \mathrm{N}$ and $185 \mu \mathrm{N}$ were applied in a twelve-cycle test. The measured lateral loads were plotted on the left $\mathrm{Y}$-axis. During the wear passes, the kinetic friction coefficient remained relatively constant near 0.1. Substantial dislocation plasticity was observed as shown in Fig. 2. Each pass was observed to nucleate more dislocations which were arranged in a symmetric array along the wear path. 


\section{References:}

[1] E Hintsala et al., JOM 69 (2017), p. 51.

[2] I Jenei and F Dassenoy, Tribology Letters 65 (2017), p. 8.

[3] S Bhowmick et al., Microsc. Microanal. 24 (2018), p. 1934.

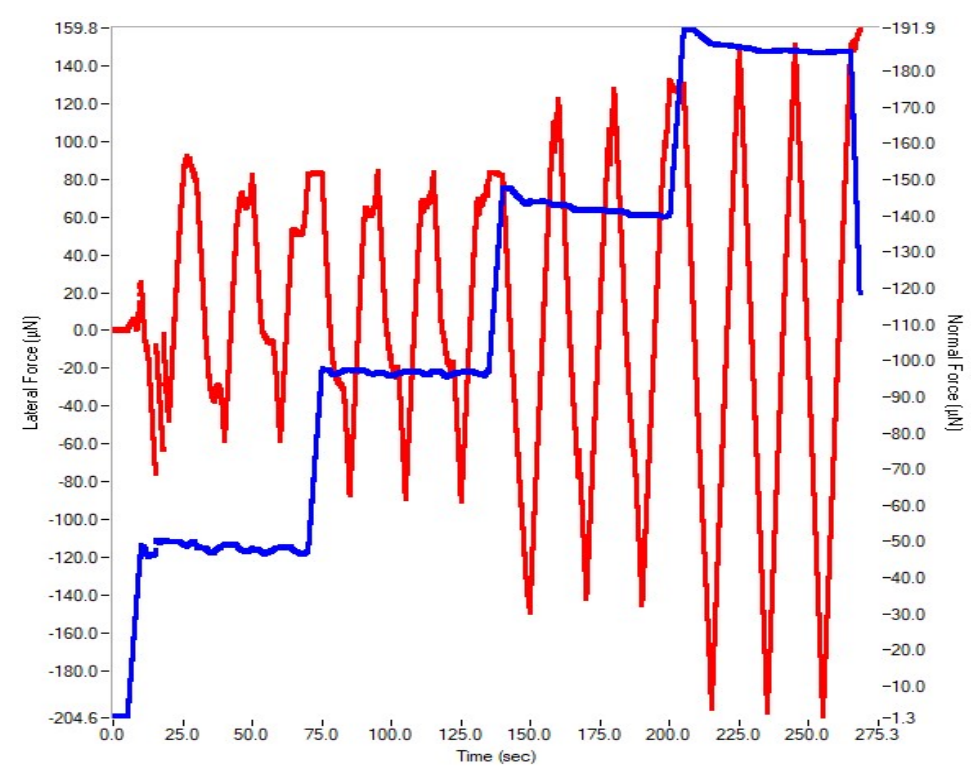

Figure 1. A fixed normal force was applied for three consecutive passes and then increased. A total 12 passes were made during the test.
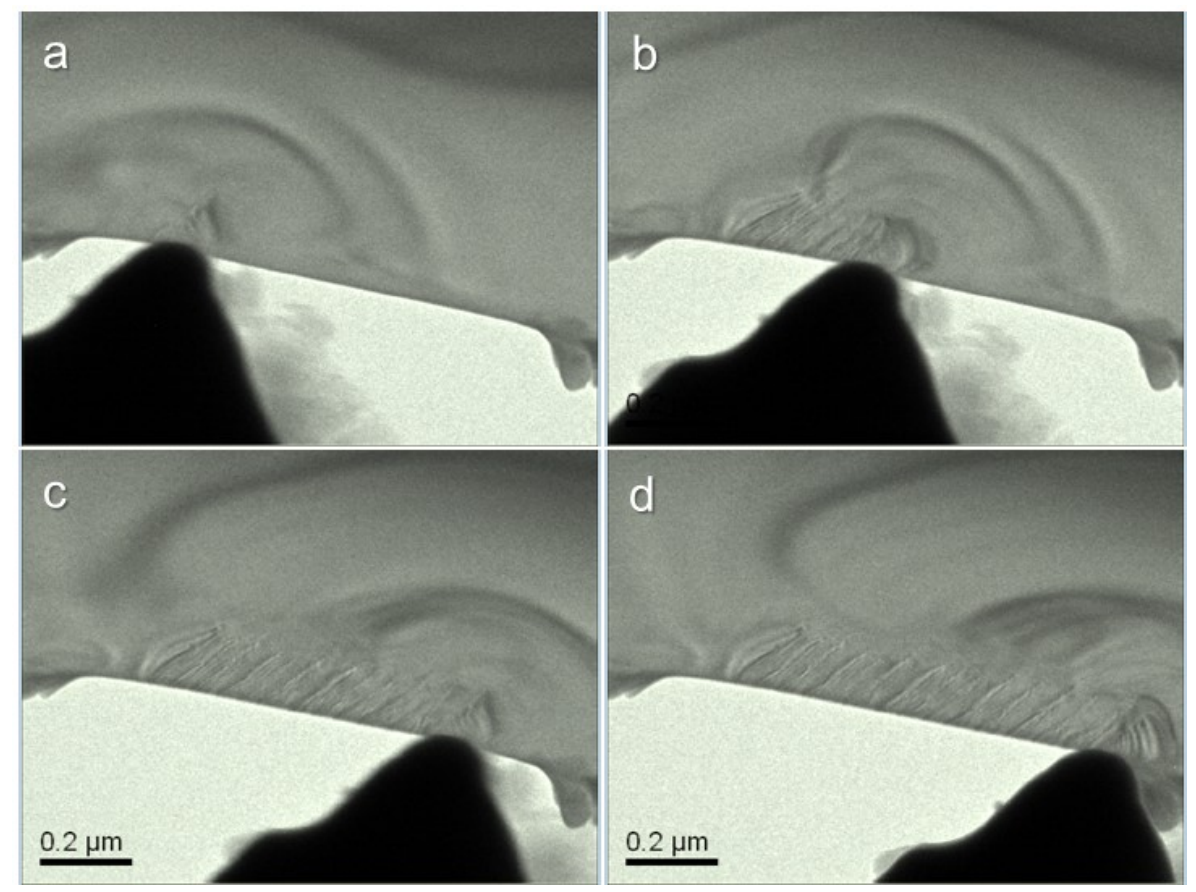

Figure 2. Development of symmetric array of defects along the wear path in olivine sample after (a) 3 rd pass (b) $6^{\text {th }}$ pass, (c) $9^{\text {th }}$ pass and (d) $12^{\text {th }}$ pass. 